\title{
Inequality Theorem regarding the lines joining corresponding vertices of two equilateral, or directly similar, triangles.
}

By Charles Tweedie, M.A., B.Sc.

$\$ 1$. Theorem.--If $\mathrm{ABC}, \mathrm{A}^{\prime} \mathrm{B}^{\prime} \mathrm{C}^{\prime}$ are any two equilateral triangles in a plane, their vertices being taken in the same sense of rotation, of the three lines $\mathrm{AA}^{\prime}, \mathrm{BB}^{\prime}, \mathrm{CC}^{\prime}$, the sum of any two is not less than the third.

Let $\mathrm{BC}$ and $\mathrm{B}^{\prime} \mathrm{C}^{\prime}$ meet in $\mathrm{P}, \mathrm{CA}$ and $\mathrm{C}^{\prime} \mathrm{A}^{\prime}$ in $\mathrm{Q}, \mathrm{AB}$ and $\mathrm{A}^{\prime} \mathrm{B}^{\prime}$ in $R$ (Fig. 2). Let $a$ be the angle between corresponding sides. Then, in virtue of the common angle $a$, the quadrilaterals

$A A^{\prime} Q R, B B^{\prime} P R, C^{\prime} P Q$ are cyclic.

Hence $\quad \mathrm{AA}^{\prime} / \mathrm{QR}=\mathrm{BB}^{\prime} / \mathrm{PR}=\mathrm{CC}^{\prime} / \mathrm{PQ}=\sin \alpha / \sin 60^{\circ}$.

i.e., $\mathrm{AA}^{\prime}, \mathrm{BB}^{\prime}, \mathrm{CC}^{\prime}$ are proportional to the sides of the triangle $\mathrm{PQR}$. $\therefore$ etc. Q.E.D.

Cor. 1. Since the triangles are equilateral we may make $\mathbf{B}^{\prime}$ or $\mathbf{C}^{\prime}$ correspond to $A$, and the theorem therefore applies to

$$
\begin{array}{lll}
\mathbf{A A} \mathbf{A}^{\prime}, & \mathbf{B B} & \mathbf{C C ^ { \prime }} ; \\
\mathbf{A B}, & \mathbf{B C}, & \mathbf{C A}^{\prime} ; \\
\mathbf{A C}, & \mathbf{B A} \mathbf{A}^{\prime}, & \mathbf{C B}^{\prime},
\end{array}
$$

Cor. 2. If the triangle $A^{\prime} B^{\prime} C^{\prime}$ become infinitesimal the theorem still applies; or, if $P$ be any point in the plane of an equilateral triangle $\mathrm{ABC}$, of the three lines $\mathrm{PA}, \mathrm{PB}, \mathrm{PC}$, the sum of any two is not less than the third.

§2. If, instead of being equilateral, the triangles $A B C$ and $A^{\prime} B^{\prime} C^{\prime}$ are only directly similar, the same construction as before leads to the relations

$$
\begin{aligned}
& \mathrm{AA}^{\prime} / \mathrm{QR}=\sin \alpha / \sin \mathrm{A}, \\
& \mathrm{BB}^{\prime} / \mathrm{PR}=\sin \alpha / \sin B, \\
& \mathrm{CO}^{\prime} / \mathrm{PQ}=\sin \alpha / \sin C .
\end{aligned}
$$


Hence $\quad A^{\prime}{ }^{\prime} \sin A, \quad B B^{\prime} \sin B, \quad C^{\prime} \sin C$,

and therefore $a \mathrm{AA}^{\prime}, b \mathrm{BB}^{\prime}, c \mathrm{CC}^{\prime}$, are proportional to the sides of the triangle PQR.

In particular, if $P$ is a point in the plane of triangle $A B C$, then $a \mathrm{PA}, b \mathrm{~PB}, c \mathrm{PC}$ are proportional to the sides of a triangle.

\$3. The case of equality of one of the quantities in question to the sum of the other two will in general only arise when $P, Q, R$ are collinear points. Now, when two triangles are directly similar, a point $O$ can be found in the plane such that, by rotation and similarity transformation, the one triangle can be transformed into the other.

When the triangles are also in perspective, the point $O$ is common to the two circumcircles of $\mathrm{ABC}$ and $\mathrm{A}^{\prime} \mathrm{B}^{\prime} \mathrm{C}^{\prime}$.*

For, let the circles $\mathrm{BRPB}^{\prime}, \mathrm{CQPC}$ cut in $\mathrm{O}$ (Fig. 3 ).

$$
\begin{aligned}
\therefore \quad \angle \mathrm{BOP}+\angle \mathrm{BRP} & =\pi, \\
\angle \mathrm{COP}+\angle \mathrm{CQP} & =\pi .
\end{aligned}
$$

Therefore $\quad \angle B O P-\angle C O P=\angle C Q P-\angle B R P$,

$$
\therefore \angle \mathrm{BOC}=\angle \mathrm{RAQ}=\angle \mathrm{BAC} \text {, }
$$

i.e., $\mathrm{O}$ is on the circle round $\mathrm{ABC}$, and $\therefore$ also on the circle $\mathrm{A}^{\prime} \mathrm{B}^{\prime} \mathrm{C}^{\prime}$.

Let, as before, $a$ be the angle between corresponding sides. If $\alpha$ is kept fixed, the angle $\mathrm{AOA}^{\prime}$ is constant. Also $\mathrm{OA} / \mathrm{OA}^{\prime}$ is constant. Hence when $\triangle A B C$ is kept fixed, and $\triangle A^{\prime} B^{\prime} C^{\prime}$ moves so that the case of equality arises, the loci of $A^{\prime}, B^{\prime}, C^{\prime}$ are equal circles through $\mathrm{A}, \mathrm{B}, \mathrm{C}$ respectively. These circles vary when $a$ is changed. Also, when $A^{\prime} B^{\prime} C^{\prime}$ reduces to a point $P$, there is equality of one of $a \mathrm{PA}, b \mathrm{~PB}, c \mathrm{PC}$ to the sum of the other two if $\mathrm{P}$ lies on the circumcircle of $\mathrm{ABC}$.

The case of two similar and similarly situated triangles may be considered as arising from $a=0$ or $a=\pi$, and the case of inequality is therefore the general case when $P, Q, R$ are at infinity. If this is not clear, a particular case of the next paragraph will render the conclusion sufficiently obvious.

- See note by Mr P. Pinkerton upon this paper, which suggested this conclusion. 
$\$ 4$. The theorem just discussed leads to an interesting analytical conclusion.

If $\mathrm{X}, \mathrm{Y}, \mathrm{Z}$ are three positive quantities, then of the three quantities

$$
\mathbf{X}+\mathbf{Y}-\mathbf{Z}, \quad \mathbf{X}-\mathbf{Y}+\mathbf{Z}, \quad-\mathbf{X}+\mathbf{Y}+\mathbf{Z},
$$

two are certainly positive; and if $X$ be the greatest, there is only uncertainty as to the sign of $Y+Z-X$. Hence the sign of $\mathbf{Y}+\mathrm{Z}-\mathrm{X}$ is the same as that of $(\mathrm{X}+\mathrm{Y}+\mathrm{Z}) \Pi(\mathbf{X}+\mathbf{Y}-\mathbf{Z})$, i.e., of $2 \Sigma \mathrm{Y}^{2} Z^{2}-\Sigma \mathrm{X}^{4}$, and if this expression is always positive, so is each of the quantities $\mathrm{X}+\mathrm{Y}-\mathrm{Z}$, etc.

Consider the coordinates of the vertices of two directly similar triangles $\mathrm{ABC}$ and $\mathrm{A}^{\prime} \mathrm{B}^{\prime} \mathrm{C}^{\prime}$ to be fixed as follows. Let $(\xi, \eta)$ denote the vertex $\mathrm{C}, a$ the inclination of $\mathrm{CB}$ to the $x$-axis, $\theta$ the angle BCA, $a$ and $b$ the sides CB and CA. Let $(x, y), \beta, \theta, \rho a$ and $\rho b$, be the corresponding elements for $\mathbf{A}^{\prime} \mathbf{B}^{\prime} \mathbf{C}^{\prime}$.

If $\mathrm{ABC}$ be fixed, and $\rho$ and $\beta$ fixed, then the equation

$$
a \mathrm{AA}^{\prime}+b \mathrm{BB}^{\prime}-c \mathrm{CO}^{\prime}=0
$$

would in general lead to a locus for $\mathrm{C}^{\prime}$ which is a bicircular quartic, which should divide the plane into regions for which

$$
a \mathrm{AA}^{\prime}+b \mathrm{BB}^{\prime}>c \mathrm{CC}^{\prime} \text {, and } a \mathrm{AA}^{\prime}+b \mathrm{BB}^{\prime}<c \mathrm{CC}^{\prime} \text {. }
$$

But we already know that the latter case can not arise. Hence the locus must, if real and finite both ways, be represented by the square of a quadratic function of $x$ and $y$, and therefore of a circular function of $x$ and $y$, or of a quadratic point function multiplied by the square of a linear function of $x$ and $y$. So long as $a, b$, and $\theta$ are distinct from zero the latter case can not arise. We are therefore led to expect that the analytic expression of the fact that the sum of any two of $a \mathrm{AA}^{\prime}, b \mathrm{BB}^{\prime}, c \mathrm{CC}^{\prime}$ is not less than the third leads to the conclusion that the expression $2 \Sigma b^{2} c^{2} \mathrm{BB}^{\prime 2} . \mathrm{CC}^{\prime 2}-\Sigma a^{4} \mathrm{AA}^{\prime 4}$ when represented in terms of $x, y$, etc., is the square of a circular quadratic function of $x$ and $y$.

The coordinates of $\mathrm{C}, \mathrm{B}, \mathrm{A}$ are

$(\xi, \eta) ; \quad(\xi+a \cos a, \eta+a \sin \alpha) ; \quad(\xi+b \cos \overline{\theta+a}, \eta+b \sin \overline{\theta+a})$. 
The coordinates of $\mathbf{C}^{\prime}, \mathrm{B}^{\prime}, \mathrm{A}^{\prime}$ are

$(x, y) ;(x+\rho a \cos \beta, y+\rho a \sin \beta) ;(x+\rho b \cos \overline{\theta+\beta}, y+\rho b \sin \overline{\theta+\beta})$.

$\therefore \quad \mathbf{A A}^{\prime 2}=(x-\xi)^{2}+(y-\eta)^{2}$,

$\mathrm{BB}^{\prime 2}=(x-\xi+\rho a \cos \beta-a \cos \alpha)^{2}+(y-\eta+\rho a \sin \beta-a \sin \alpha)^{2}$,

$\mathrm{CC}^{\prime 2}=(x-\xi+\rho b \cos \overline{\theta+\beta}-b \cos \bar{\theta}+a)^{2}+(y-\eta+\rho b \sin \overline{\theta+\beta}-b \sin \overline{\theta+a})^{2}$.

The expression

$$
2 \Sigma b^{2} c^{2} \mathrm{BB}^{\prime 2} \cdot \mathrm{CC}^{\prime 2}-\Sigma a^{4} \mathrm{AA}^{\prime 4}
$$

may then be proved equal to

$$
\begin{gathered}
4 a^{2} b^{2}\left\{\left(\overline{x-\xi^{2}}+\overline{y-\eta^{2}}\right) \sin \theta-(x-\xi)(a \sin \overline{\theta i a}-b \sin \alpha-\rho a \sin \overline{\theta+\beta}+\rho b \sin \beta)\right. \\
+(y-\eta)(a \cos \overline{\theta+a}-b \cos \alpha-\rho a \cos \overline{\theta+\beta}+\rho b \cos \beta)\}^{2} .
\end{gathered}
$$

This result naturally includes the whole of the preceding theory. The labour of verification may be simplified by writing $\mathrm{X}$ for $x-\xi$ and $Y$ for $y-\eta$, and replacing $c^{2}$ wherever it occurs by its equivalent $a^{2}+b^{2}-2 a b \cos \theta$.

By taking $a=0, \beta=0$ or $\pi$, we obtain the case of figures similar and similarly situated, and there is equality for $\left(\overline{x-\xi^{2}}+\overline{y-\eta^{2}}\right) \sin \theta-(x-\xi) a \sin \theta(1 \pm \rho)+(y-\eta)(a \cos \theta-b)(1 \pm \rho)=0$. Hence if the sides of two congruent triangles $(\rho=1)$ are like directed there can only be equality when $(x-\xi)^{2}+(y-\eta)^{2}=0$, i.e., when two corresponding vertices are coincident. In all other cases the inequality holds good.

\$5. The inequality theorem admits of extension to two similar triangles in parallel planes, when there is no case of equality, so long as the planes are distinct.

The most interesting case, that of two equilateral triangles, is also the simplest to discuss.

Let the distance between the two planes be $d$ and project $\mathrm{A}^{\prime} \mathrm{B}^{\prime} \mathrm{C}^{\prime}$ upon the plane of $A B C$ into $A_{1} B_{1} C_{1}$.

Let $a, \beta, \gamma$ denote $\mathbf{A A}^{\prime}, \mathbf{B B}^{\prime}, \mathbf{C C}^{\prime}$, and $a_{1}, \beta_{1}, \gamma_{1} \quad \mathrm{AA}_{1}, \mathrm{BB}_{1}, \mathrm{CC}_{1}$. 


$$
\begin{aligned}
& \therefore \quad a^{2}=d^{2}+a_{1}^{2} ; \quad \beta^{2}=d^{2}+\beta_{1}^{2} ; \quad \gamma^{2}=d^{2}+\gamma_{1}{ }^{2} . \\
& \therefore \quad 2 \Sigma \beta^{2} \gamma^{2}-\Sigma a^{4}=2 \Sigma\left(d^{2}+\beta_{1}^{2}\right)\left(d^{2}+\gamma_{1}^{2}\right)-\Sigma\left(d^{2}+a_{1}^{2}\right)^{2} \\
& =3 d^{4}+2 d^{2} \Sigma a_{1}{ }^{2}+\left(2 \Sigma \beta_{1}^{2} \gamma_{1}{ }^{2}-\Sigma a_{1}{ }^{4}\right) \\
& =\text { a sum of positive quantities, } \quad \therefore \text { etc. }
\end{aligned}
$$

In the case of directly similar triangles we must prove

$$
\begin{gathered}
2 \Sigma b^{2} c^{2}\left(d^{2}+\beta_{1}^{2}\right)\left(d^{2}+\gamma_{1}^{2}\right)-\Sigma a^{4}\left(d^{2}+a_{1}^{2}\right)^{2}>0 \\
\text { i.e., } \quad d^{4}\left(2 \Sigma b^{2} c^{2}-\Sigma a^{4}\right)+2 d^{2}\left\{\Sigma b^{2} c^{2}\left(\beta_{1}{ }^{2}+\gamma_{1}{ }^{2}\right)-\Sigma a^{4} a_{1}{ }^{2}\right\} \\
+\left(2 \Sigma b^{2} c^{2} \beta_{1}^{2} \gamma_{1}^{2}-\Sigma a^{4} a_{1}^{4}\right)>0 .
\end{gathered}
$$

The first and third members of this inequality are already positive and the theorem is true provided

$$
\begin{aligned}
\Sigma b^{2} c^{2}\left(\beta_{1}^{2}+\gamma_{1}^{2}\right)-\Sigma a^{4} a_{1}^{2} & >0, \\
\text { i.e., } \Sigma a^{2} a_{1}{ }^{2}\left(b^{2}+c^{2}-a^{2}\right) & >0 \\
\text { or } \Sigma a^{2} a_{1}{ }^{2} \cdot 2 b c \cos \mathrm{A} & >0, \\
\text { i.e., } a b c \Sigma a \cos \mathrm{A} a_{1}{ }^{2} & >0 \\
\text { or } \Sigma{a_{1}}^{2} \sin 2 \mathrm{~A} & >0 .
\end{aligned}
$$

The last statement is obvious for an acute-angled triangle. For a general proof, determine $O$ and $O_{1}$ the circumcentres of the t.wo triangles. Join $\mathrm{OO}_{1}$, and the centres to the corresponding vertices.

Let $\mathrm{OA}$ make an angle $\psi$ with $\overrightarrow{\mathrm{OO}}_{1}$, and $\mathrm{OA}_{1}$ an angle $\psi_{1}$ with $\overrightarrow{\mathrm{OO}}_{1}$. Then if $r$ and $r_{1}$ be the radii and $d$ the distance $O O_{1}$, we have

$$
\mathrm{AA}_{1}^{2}=d^{2}+r^{2}+r_{1}^{2}-2 r r_{1} \cos \left(\psi-\psi_{1}\right)-2 d\left(r \cos \psi-r_{1} \cos \psi_{1}\right) \text {. }
$$

To get $\mathrm{BB}_{1}^{2}$ put $\psi+2 \mathrm{C}$ for $\psi$ and $\psi_{1}+2 \mathrm{C}$ for $\psi_{1}$;

$\therefore \mathrm{BB}_{1}^{2}=d^{2}+r^{2}+r_{1}^{2}-2 r r_{1} \cos \left(\psi-\psi_{1}\right)-2 d\left(r \cos \overline{\psi+2 \mathrm{C}}-r_{1} \cos \overline{\psi_{1}+2 \mathrm{C}}\right)$.

Similarly $\mathbf{C C}_{1}^{2}$ is found by adding $\mathbf{2 C}+2 \mathrm{~A}$ to $\psi$ and $\psi_{1}$.

Hence $\Sigma a_{1}^{2} \sin 2 \mathrm{~A}=\left(d^{2}+r^{2}+r_{1}^{2}-2 r r_{1} \cos \overline{\psi-\psi_{2}}\right) \Sigma \sin 2 \mathrm{~A}+0$,

(for the cofficients of $d r$ and $d r^{\prime}$ are zero)

$$
=\left(d^{2}+r^{2}+r_{1}^{2}-2 r r_{1} \cos \overline{\psi-\psi_{1}}\right) \times 4 \sin A \sin B \sin C,
$$

and $\therefore \Sigma a_{1}^{2} \sin 2 A$ is always positive. $\therefore$ etc. Q.E.D. 